\title{
Value Added Service Management in 3G networks
}

\author{
N. Houssos, E. Gazis and \\ S.Panagiotakis, \\ Communication Networks Laboratory, \\ Department of Informatics and \\ Telecommunications, \\ University of Athens \\ 15126 Athens \\ Greece \\ Sandra Quesnel \\ Thales Communications \\ 66, rue du fosse blanc \\ 92231 Gennevilliers \\ France
}

\author{
S. Gessler and A. Schuelke, \\ NEC Europe Network Laboratories \\ Adenauerplatz 6 \\ 69115 Heidelberg \\ Germany
}

\begin{abstract}
3rd generation mobile communication networks (3G) have been heralded as a paradigm shift that will irreversibly change the structure of the telecommunications industry. In an ideal "3G world", roaming users will be offered an abundance of value-added services typically developed by independent service providers. It is commonly recognized that the plethora of business combinations and technical implementations in the emerging $3 \mathrm{G}$ era will substantially raise the bar on the respective management frameworks. The present contribution deals with service provision aspects in $3 \mathrm{G}$ network environments and particularly focuses on service deployment and management matters. We conduct a requirement analysis of the $3 \mathrm{G}$ service provision process and illustrate the need for an integrated service management platform. Next, we elaborate on the architectural and implementation details of a service provision management platform capable of intelligently delivering downloadable applications to $3 \mathrm{G}$ mobile users, as designed and developed within the frame of the EC IST project MOBIVAS. Finally, we conclude the paper by positioning our approach with regard to relevant standardization activities and laying out directions for future work.
\end{abstract}

\section{Keywords}

Service management, value-added services, $3 \mathrm{G}$ networks. 


\section{Introduction}

The forthcoming era of $3^{\text {rd }}$ generation (3G) mobile communications is heralded to change the wireless telecommunication experience dramatically. The development of $3 \mathrm{G}$ systems will be a major step towards the convergence of the telecommunications and the Internet industries and will result in the creation of an open and highly competitive network services marketplace, where $3^{\text {rd }}$ party service developers, often termed Value-Added Service Providers (VASPs), will be able to market their services to users of wireless and wired networks in a globally unified way ${ }^{1}$. Thus, the efficient delivery of applications to end users will be more complex than ever before, bearing also in mind the growing demand for ubiquitous, personalised and terminal aware service provision [1]. In this context, the task of service management becomes significantly more demanding, as well as critical for the continuous growth and market success of the converging worlds of telecommunications and the Internet. This paper proposes a potential solution to this issue, by introducing a distributed software framework for the efficient management and intelligent provision of value added services in $3 \mathrm{G}$ networks.

The rest of the document is organized as follows: at first, we discuss the substantial implications that the arrival of $3^{\text {d }}$ generation systems is expected to have on service provision to mobile users and the relevant emerging need for sophisticated service management platforms. In Section 3, we present the architecture, functionality and implementation aspects of a software framework that aims to address this need, with particular focus on its main component, namely the Value Added Service Manager (VASM). Subsequently, example operations in the MOBIVAS framework are presented in the form of sequence diagrams. In section 5, we explain how such a platform complements and adds value to relevant standardisation and research activities of various organisations. The last sections of this paper are dedicated to summary, conclusions, and acknowledgments.

\section{Service provision in $3 G$ networks}

\subsection{The emerging era of 3G}

In mobile telecommunication environments until $2 \mathrm{G}$, network access and service/application provision has been statically bundled together in various subscription-based offerings by network operators. The introduction of $3 \mathrm{G}$ systems [7], such as UMTS, which distinguish between the network and the service/application domain, renders such strategies inefficient. To "make 3G happen" a critical mass of applications and services that are novel, attractive and

\footnotetext{
${ }^{1}$ In a $3 \mathrm{G}$ context the term service and application are often used interchangeably to refer to an information technology product whose value resides mostly in functionality and content, rather than transport or connectivity, much like the vast majority of applications that are available nowadays through the Internet.
} 
exploit the unique capabilities of $3 \mathrm{G}$ networks ${ }^{2}$ to the benefit of the user's experience is necessary [2][3]. In contrast to the prevailing $2 \mathrm{G}$ norm, where services are developed by a limited number of telecommunications experts (e.g., operators, equipment vendors), $3 \mathrm{G}$ applications should be substantially contributed by a large variety of non-telecomm literate market players, including software vendors and content providers. This fact is expected to foster competition and differentiated service offerings and to accelerate system deployment and service take-up, thus minimizing investment risks. It is therefore envisaged that the evolution of mobile telecommunications towards $3 \mathrm{G}$ - and beyond it - will be driven by two major forces:

- A multitude of services with rich functionality that will provide the revenue stream required to amortize the high $3 \mathrm{G}$ investments.

- The existence of multiple business players in the value chain that will cooperate in the provision of enriched service offerings.

\subsection{The need for integrated service management platforms}

In the context described in the previous paragraph, there is a shift in the traditional paradigms of management from managing network elements and networks to managing services. The abundance of services [4] [5] that will be typically developed by many co-operating entities and will need to be rapidly deployed on multiple types of networks [6] significantly complicates service deployment and provisioning compared to $2 \mathrm{G}$ networks. Moreover, the requirement for QoS provisioning and for sophisticated, flexible billing schemes makes the service management task more difficult than it is in the Internet world. Therefore, a need is emerging for intelligent and flexible service provision platforms that will mediate between service developers, network operators and end-users and provide a set of functionality not available until now.

In the rest of this section, we present and discuss certain features that we consider necessary for a platform supporting deployment and management of services in next-generation mobile communication networks. The main features we have identified are the following:

\subsubsection{Dynamic, on-line, generic deployment and management of VASs}

To be made globally available, $3 \mathrm{G}$ services - typically - developed by independent application providers, must adhere to a deployment model that is network and terminal independent. Straightforward and automated deployment procedures will be a critical feature for the successful lifecycle management of $3 \mathrm{G}$ services. Its significance lies in the fact that it enables marketing a large number of services whilst reducing service time-to-market, two of the most important business requirements of $3^{\text {rd }}$ generation networks. The service deployment process unfolds itself into a number of distinct procedures:

\footnotetext{
${ }^{2}$ These include transparent mobility management, location reporting, identity authentication, information ciphering, paging, to name but a few.
} 
- Initial contact and business level agreement: The independent application provider negotiates the details of its business collaboration with the other market players contributing to the $3 \mathrm{G}$ service offering (e.g. the $3 \mathrm{G}$ mobile network operator), a process that will typically result in a formal contract.

- Service management: Service management procedures deal with the initial registration of a new service and the instantiation of the respective service profile, the application of subsequent modifications and updates to it and, finally, the deregistration of the service and the deletion of its profile. Given that these services may require transport services with specific characteristics (e.g. QoS, free-ofcharge bearers), a particular set of network management procedures must be able to execute automatically, in the context of the aforementioned service management procedure. For example, service upgrade may require introduction of different tariffing schemes and modification of QoS provisioning policies. The necessary actions for any of the operations described above should take place in a single operation that should be performed on-line and typically last for no more than a few seconds, without interrupting service provision, namely the user service access experience. Example use cases for service deployment by a $3^{\text {rd }}$ party are depicted in Figure 1.

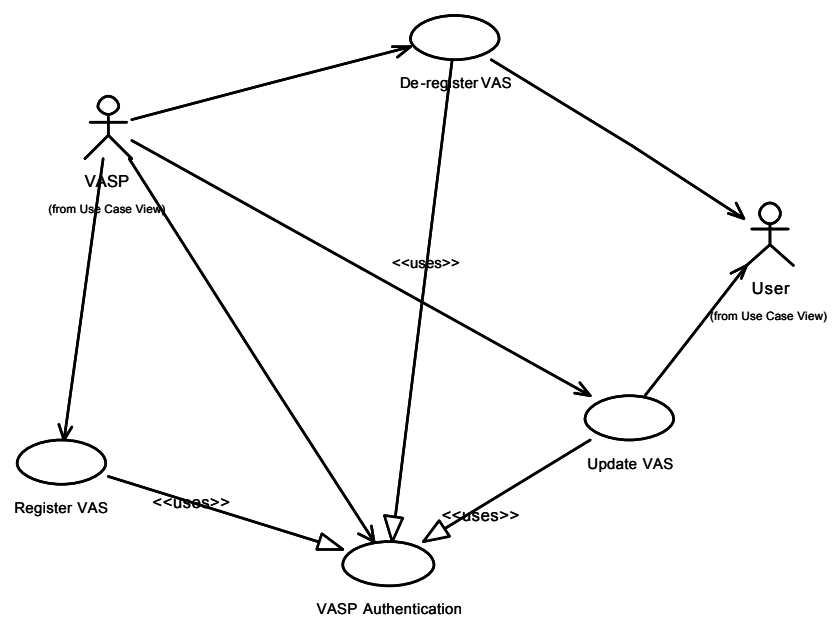

Figure 1. Use cases for VAS deployment by a $3^{\text {rd }}$ party.

\subsubsection{Intelligent service discovery}

Given that in $3 \mathrm{G}$ there is no prior relationship between service/application providers and mobile users, the need for a unified service discovery mechanism arises. Taking into consideration the huge range of services available to the mobile users of the forthcoming $3 \mathrm{G}$ networks and the competitive nature of the new era, the need for an intelligent, efficient, and intuitive mechanism for personalized 
service discovery and provision to mobile subscribers is widely recognized. In addition to that, users are expected to access services from a wide variety of terminals with highly varying characteristics. Ideally, the service discovery process should be context-aware, where the context includes user privacy preferences and location, mobile terminal and network capabilities as well as application features and requirements. The implementation of such schemes requires the application of flexible terminal capability representation format and announcement procedures, like the one adopted by the 3GPP MExE specification [8], as well as employing sophisticated user profiling mechanisms. Example use cases for service discovery by a $3^{\text {rd }}$ party are depicted in Figure 2.

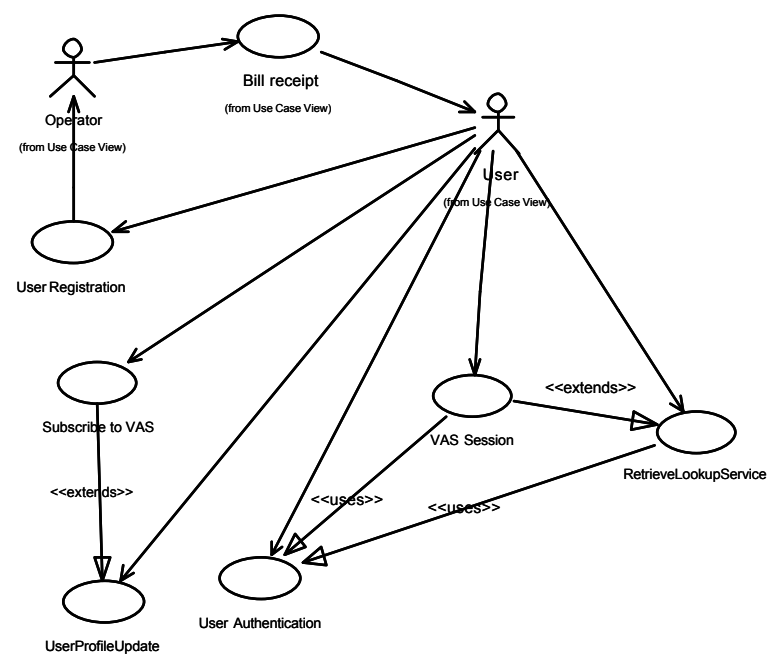

Figure 2. Use cases for service discovery by a $3^{\text {rd }}$ party.

\subsubsection{Flexible and dynamic billing}

The demarcation between the network and the service/application domains that has been explicitly architected in 3G systems implies that the respective charging and billing architecture should portrait similar - if not greater - flexibility in its design. As a minimum requirement, network-related pricing models and policies should be completely independent from service-related ones, with regard to both formulation and application matters. The development of innovative $3 \mathrm{G}$ applications is expected to further raise the bar on candidate charging \& billing architectures by requiring support for advanced and sophisticated features (e.g. pre-paid, hotbilling, reverse charging, etc). In addition, the service provision process must interact dynamically with the charging process in order to provide notification mechanisms that communicate to the user the portion of the overall charges that attributes to each domain (i.e. network or service). Furthermore, such pricing information must be provided in a format that is clear, succinct and readily understandable by non IT-literate users. 
It is doubtful if traditional management paradigms can satisfy these diverse requirements which may span across multiple administrative domains, e.g. in a global roaming scenario. An attractive approach for meeting these requirements is by means of an integrated service provision and management platform.

\section{MOBIVAS - A flexible platform for dynamic service provisioning}

\subsection{Overview and architecture}

In the frame of the MOBIVAS project, a distributed software platform is introduced, aiming to provide an advanced and flexible solution for the deployment and management of value-added services offered to $3 \mathrm{G}$ mobile users. The entire framework comprises several components, which are vertically placed within the administrative domains of virtually all the business entities that can be involved in VAS provision.

The functionality of each of the MOBIVAS components can be described as follows:

- The VASM is the central component of the platform, catering for the automatic service registration and deployment by $3^{\text {rd }}$ party VASPs including the necessary reconfiguration actions on the underlying network for optimal service provision. Moreover, it provides to the end user a mobile portal for personalized and terminal-aware service discovery. It is worth noting that the VASM is connected via a (public or private) IP network to the UMTS core network and can be owned either by a mobile operator or by another entity (e.g., an ISP), undertaking the role of the MOBIVAS platform operator.

- The L4+ Systems [12] are able to perform filtering of IP traffic, monitoring of specific flows and QoS provisioning, by examining transport layer information in addition to the network layer data used by conventional IP routers. In the proposed architecture the L4+ systems are located at the edge of the connectivity network of the mobile network operator so that they process all traffic between VASPs and users. They are configured by the VASM to provide QoS to specific IP traffic flows that concern active VAS sessions and produce VAS usage records that are necessary for billing.

- The Charging, Accounting and Billing (CAB) system, using the information provided by the L4+ systems and the UMTS network for the usage of its resources, calculates the overall charges induced by service consumption and apportions the aggregate revenue among VASPs, the MOBIVAS platform operator - as well as the $3 \mathrm{G}$ network operator, if different from the latter. The system produces a single, itemized user bill. It has been designed to allow the application of arbitrarily complex billing models and tariffing schemes and to be dynamically reconfigurable by the VASM. A more detailed description of the overall approach for charging and billing is beyond the scope of the present paper. A thorough treatment of relevant issues can be found in [9] [10] [11]. 


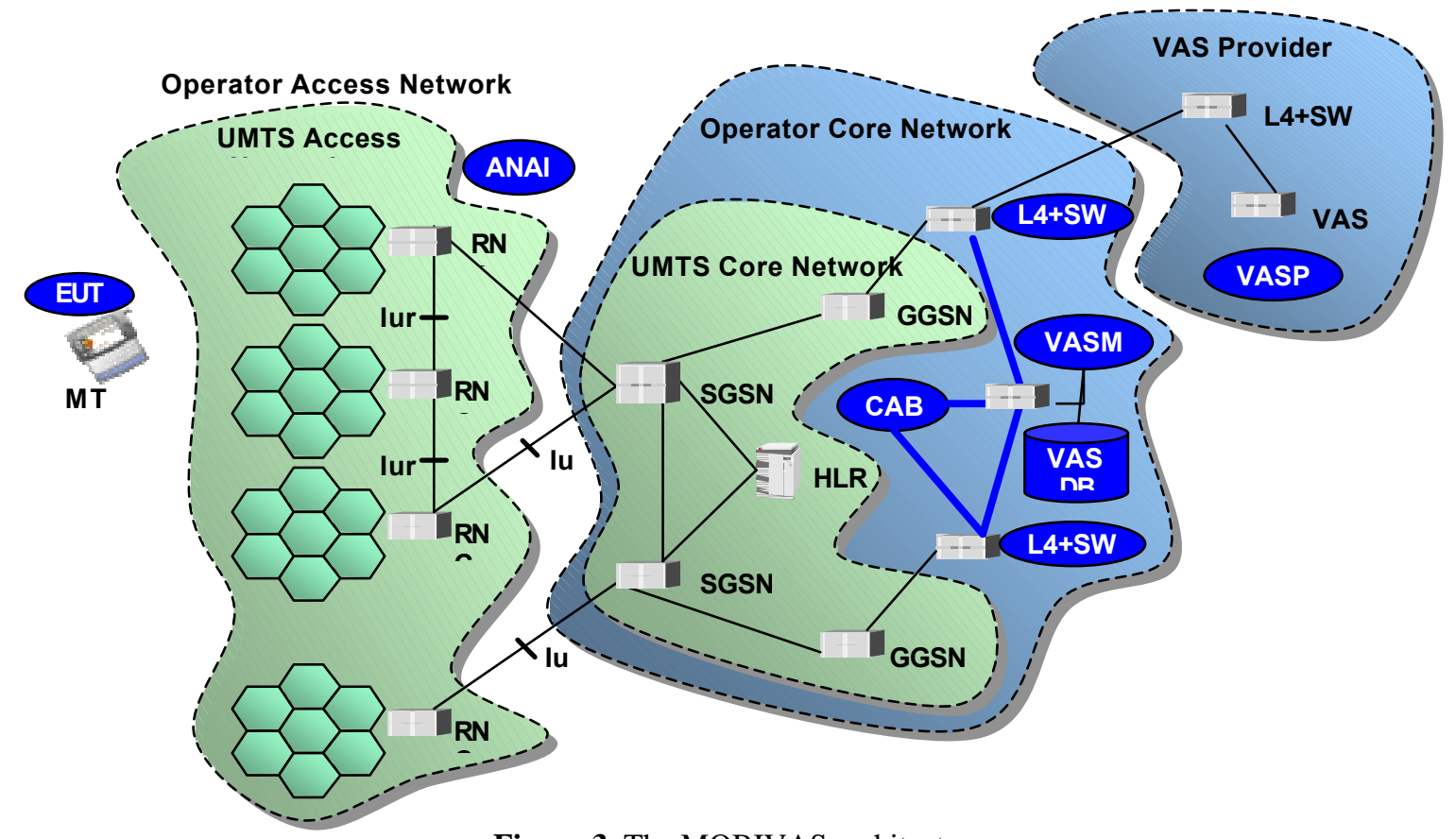

Figure 3. The MOBIVAS architecture

- The VASP component of the platform handles secure terminal access to a repository of VAS clients and also contains web interfaces to service management functionality offered by the VASM to the VASPs (e.g., VAS deployment).

- The End User Terminal Platform EUT) comprises modules that provide a variety of functionality, like a graphical user interface for VAS discovery, secure downloading and lifecycle management (e.g., start/stop/abort/resume) of VAS clients, as well as initial user registration with the platform and subsequent user login/authentication.

- The Access Network Added Intelligence (ANAI) uses the ReSoA architecture [13] to optimize the QoS at the wireless link, by taking into consideration the particular characteristics of the radio access technology used.

The platform architecture as well as an example physical placement of the abovementioned components is depicted in Figure 3. Note that while integration of certain framework parts (e.g., VASM and CAB) to a single physical location is possible, platform components can be physically distributed, since only IP connectivity is assumed to be available between them.

The introduction of the MOBIVAS framework results to the burden of service deployment and management being to a large extent moved from the VASP to the service platform operator. The platform is dynamically adaptive, so that it can manage a wide range of disparate services over a variety of underlying radio access and core transport networks. Moreover, the intelligence required to implement the 
service provision adaptation functionality is placed in the service platform, thus lowering requirements on mobile terminals and application logic and extending the applicable service provision domain. This way, the mobile terminal need not adapt to the service requirements; rather the service delivered via the download mechanisms is adapted to the mobile terminal capabilities by the platform.

This paper focuses mainly on the functionality, design and implementation of the VASM. These topics are elaborated in the subsequent sections.

\subsection{The Value-Added Service Manager (VASM)}

The Value-Added Service Manager (VASM) is the heart of the MOBIVAS system. It serves as a mediator between the interconnecting network infrastructure, the VASP network, the access network and the mobile users and coordinates the whole service provision process by appropriate controlling and configuring all the MOBIVAS components. The VASM is responsible for a number of important tasks including mechanisms for VAS deployment and data management, service discovery by end-users, VASP and user authentication and adaptation of VAS provision to user preferences and terminal characteristics.

The architecture of the VASM is depicted in Figure 4.

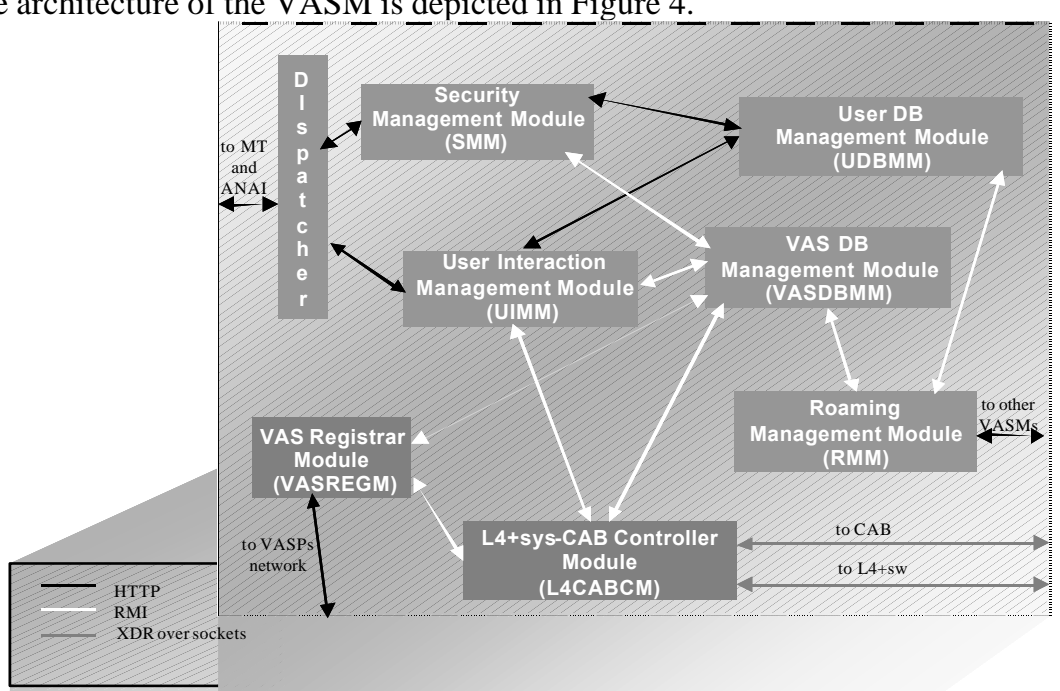

Figure 4. Architecture of the VASM component

In the following paragraphs, the functionality of the main modules of the VASM is presented.

\section{UIMM}

The User Interaction Management Module (UIMM) is the co-ordinating module of the VASM, responsible for all interactions concerning intelligent service discovery. The UIMM is responsible for presenting to the user listings of the available services, matched to the terminal capabilities, user preferences and location. To achieve this, the UIMM uses information from appropriate VAS and 
user profiles databases, as well as terminal capability and location information that is provided by the terminal.

\section{SMM}

The MOBIVAS security requirements are dealt within a SPKI infrastructure, which brings mainly the advantages of a key-oriented system, namely, a decentralized model, the flexible handling of authorization, and the power of certificate delegation.

The Security Management Module (SMM) in the VASM is responsible for :

- Secure communication between MS and VASM: MOBIVAS exchanges between MS and VASM for user authentication, terminal classification and service negotiation procedures, and secure access to core network components.

- Secure communication between VASM and VASP: MOBIVAS exchanges between VASM and VASP for VASs and VASPs registration/deregistration procedures, and secure link between the core and VASP network.

The SMM uses Authentication and Anonymity security mechanisms to preserve users privacy.

It is worth noting that the (SPKI-based) MOBIVAS security mechanisms do not require the presence of security features in the underlying protocols (e.g., HTTP, RMI). Further details regarding how security is implemented in MOBIVAS are beyond the scope of the present paper.

\section{UDBMM}

The User Database Management Module (UDBMM) handles necessary profile information about and from the user. In more specific it handles five different profiles:

- The User Identification Profile: this information is typically hosted by the network operator itself in its customer database (e.g. HLR). The MOBIVAS User database (User DB) is designed as an add-on to the operator's customer database. On request the UDBMM can derive user identification information (like name, address, IMSI No, etc) directly from the operator's DB instead of hosting this data by its own. The profiles described in the remaining of the section are hosted by the UDBMM itself. For the requesting party it is transparent, whether the requested data are derived from the operator's or he MOBIVAS's profile database.

- The User Subscription Profile: This information is used to administrate subscription of users to the MOBIVAS platform.

- The User Security Profile: It consists mainly of user-specific authorization and authentication information such as password, PGP Key, Certificates etc.

- The User Preferences Profile: It stores generic, service-independent user preferences (e.g., language, preferred quality).

\section{VASREGM}

The VAS Registrar Module (VASREGM) is responsible for interacting with 3rd party service providers. Through the VASREGM the MOBIVAS operator provides VASPs with a way to automatically deploy their services. Based on an XMLencoded VAS descriptor, the VASREGM co-ordinates service deployment, 
including any required reconfiguration actions (e.g., in the L4+ or CAB systems). The service provider is able to manage (add/delete/update) its services via a convenient web-based interface.

\section{LACABCM}

The L4+ sys and CAB Controller Module (L4CABCM) undertakes the configuration management of the $\mathrm{CAB}$ system and the network border routers termed L4+ systems.

The configuration of L4+ systems with traffic classes and metering policies follows a push pattern, where the VASM proactively provisions the appropriate configuration information in the context of the VAS management procedures. In policy-based management parlance, the VASM acts as a Policy Decision Point, while the L4+ systems act as Policy Enforcement Points, much like in [15] ${ }^{2}$.

The configuration of the $\mathrm{CAB}$ system concerns the tariff information required to transform resource usage records to billing and accounting records within the CAB. The CAB system will dynamically query the VASM for the tariff information of a particular service, thus the interaction follows a pull pattern which is termed outsourcing in policy-based management parlance.

\subsection{Implementation and demonstration of the MOBIVAS prototype}

\subsubsection{MOBIVAS implementation}

The service management platform described above has already been designed and implemented.

The programming language mainly used for the overall MOBIVAS platform development was Java, since it is a ubiquitous, de-facto, industry standard that provides several highly desirable characteristics, such as code portability across hardware architectures and operating systems, built-in facilities for distributed programming and security, as well as a rich set of high-level APIs (e.g., RMI, Servlets, JDBC, etc.) that provide the ground for rapid development of sophisticated applications. Only the components having a greater degree of realtime requirements and network dependence (L4+ Sys, CAB system) were developed in $\mathrm{C}$, to optimize performance. The VASM, being an entirely network independent, non-real-time component was entirely built in Java.

The implementation approaches used for each VASM module, as well as the interprocess communication mechanisms used are depicted in Figure 4. It is obvious from the figure that communication in interfaces that span administrative boundaries (e.g., VASP-VASM, end user terminal - VASM) is based on standard, highly interoperable technologies like HTTP and XML. Java servlets, a common, java-based way of enhancing HTTP request processing with advanced logic, is used on the server-side of HTTP communication. On the contrary, the inner modules of each component communicate with each other using more efficient

\footnotetext{
${ }^{2}$ It is worth noting that the communication between L4CABCM and L4+ systems is performed by using a proprietary protocol, instead of COPS, since a COPS PEP implementation for the L4+ system is not available yet.
} 
mechanisms: for example Java RMI is used as the distributed software technology for the communication of the internal VASM modules. The VASM has been designed and implemented in a way that the various modules can be either integrated in one physical location or completely distributed, since any two IPconnected networks nodes may communicate over Java RMI. It is worth noting that pilot trials of platform operation have shown that the VASM performance is acceptable also in the case of complete distribution of its modules, even over the Internet.

\subsubsection{MOBIVAS demonstration}

The MOBIVAS prototypical framework has been tested in three small-scale pilot demonstrations and will be also demonstrated in a field trial scheduled for the first half of 2002. The pilot operations comprised esting the functionality of the platform in an Ethernet/wireless LAN environment, while the field trial is planned to be performed over a UMTS testbed, so that the platform operation in a realworld $3 \mathrm{G}$ environment is demonstrated. Figure 5 depicts the testbed topology and the physical placement of MOBIVAS components of the MOBIVAS pilot1, performed at the University of Athens.
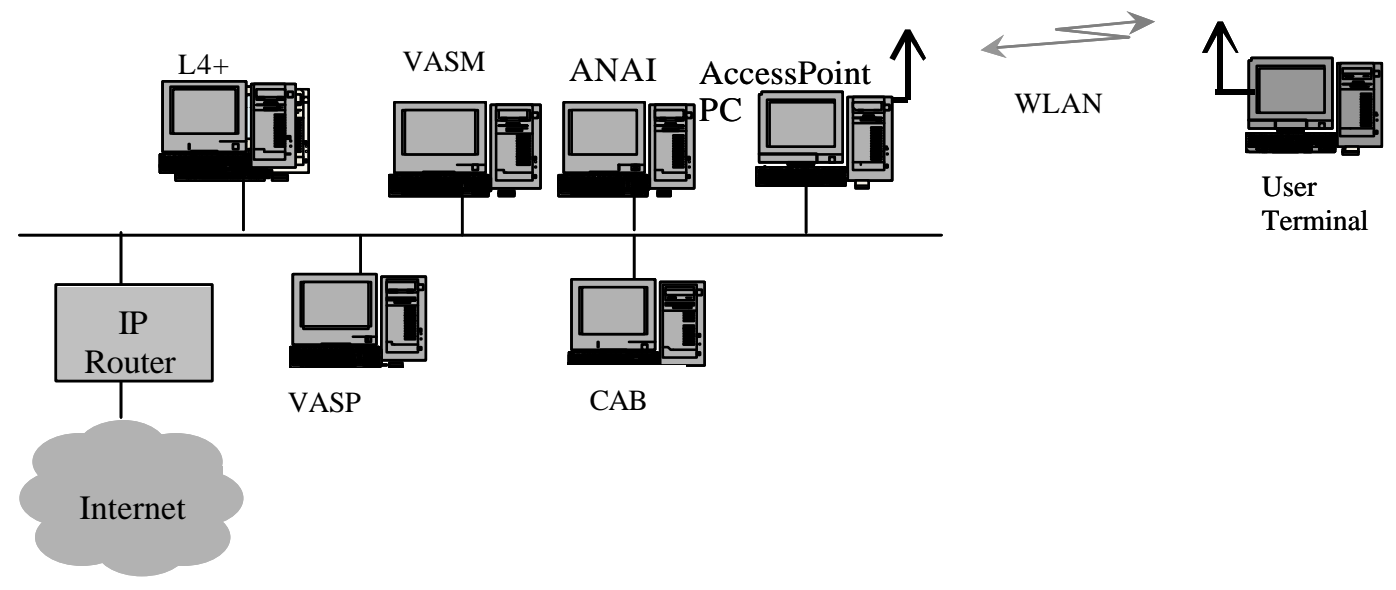

Figure 5. Example testbed topology in the MOBIVAS pilot1

\section{Example MOBIVAS operations}

\subsection{Service Deployment}

Service deployment in the presented platform follows the model introduced in section 2.2.1. The platform becomes aware of how the service should be deployed and provisioned making use of the service definition, which is provided by the VASP via an appropriate interface (e.g., web interface) in the form of an XML document, conforming to a universally common Document Type Definition 


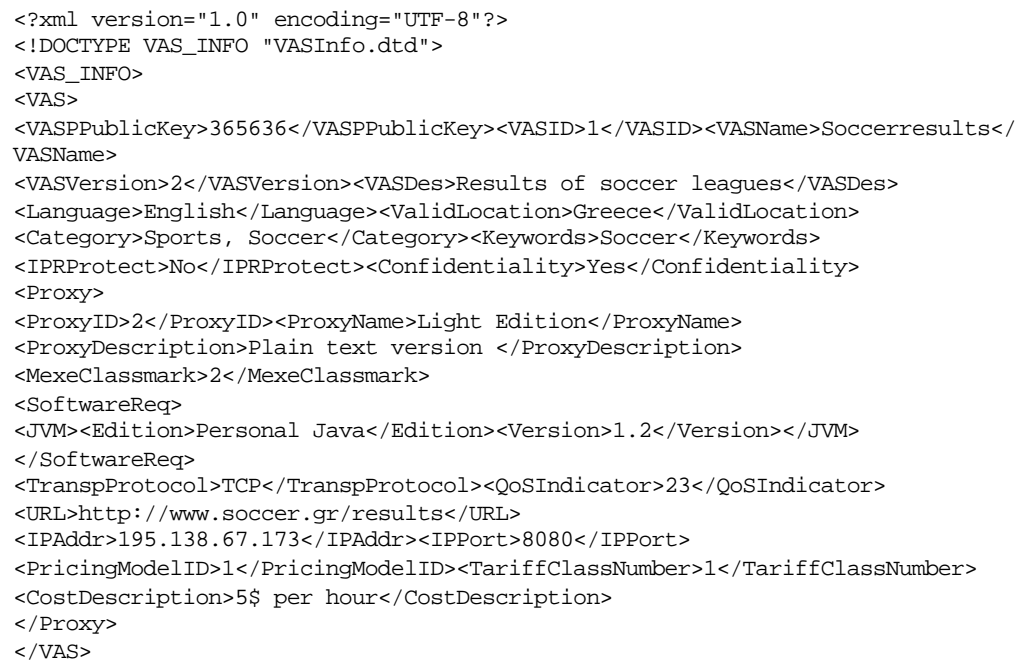

Figure 6. Example of a simplified XML VAS descriptor.

(DTD). An example of a service XML descriptor (simplified for readability) is depicted in Figure 6. The VASM first stores the appropriate service data in the VAS DB, and then performs the necessary configuration actions, which in case of our prototype comprise the configuration of the MOBIVAS provider's L4+ systems for QoS provisioning and service access accounting. When all necessary actions have been performed, the operation is considered complete, the service is made available to mobile users and the VASP receives a response message indicating the outcome of the entire operation.

The VASP is then able to subsequently remove a service from the platform or perform complex modifications of its definition (e.g., change service tariffs, insert, delete or modify individual service versions). It is worth noting that all above operations are dynamic in the sense that they are performed on-line and that overall service provision and system operation is not affected or interrupted during them. A message sequence chart with the interactions taking place for the deployment of a new service is shown in Figure 7.

\subsection{Service Discovery}

Intelligent, customised service discovery is included among the most critical postdeployment service provisioning functions offered by the MOBIVAS platform. Through a personalized, web-portal like user interface, accomplished by the service discovery client, users are provided with the ability to discover and choose the services they wish to access. The message sequence chart of Figure 8 depicts the successful case of such a service discovery session. After login/authentication, the user downloads (if not already available) the most updated version of the service discovery client supported by his/her current terminal and instructs 


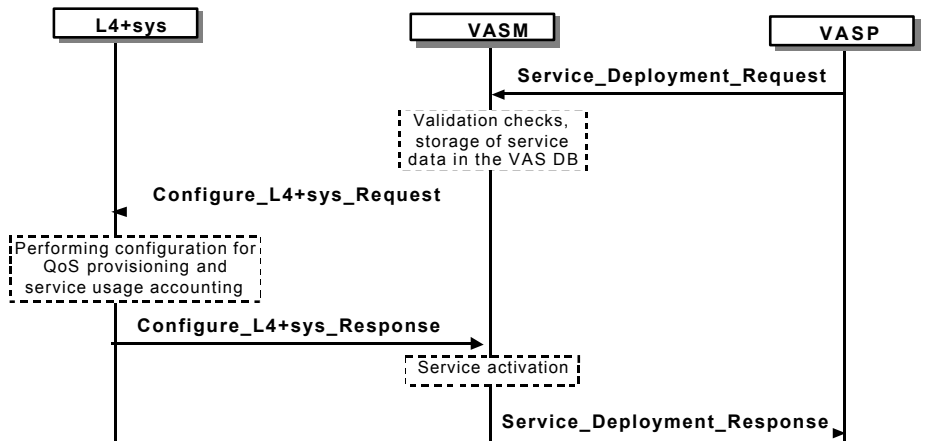

Figure 7. Message sequence diagram for the deployment of a new service

a service listing by specifying his/her desirable keywords or VAS category. The requested listing is provided to the user after filtering the VAS DB records according to the applied terminal capabilities, the user location and the user preferences specified in the user profile. The terminal capability announcement adopts the approach specified in the 3GPP MExE specification [8], which is based on the $\mathrm{W} 3 \mathrm{C}$ CC/PP standards, while user profile management is also inline with the 3 GPP specifications. Each service in the service discovery listings is associated with a short description and indicative tariffing information, so that the user may choose services based on reduced cost criteria. Following the service discovery phase the user is able to select the desired service client and to download it to his/her terminal. During service access, service usage records from L4+ systems and UMTS network's metering nodes reach the CAB, so that the appropriate charges are included in the user bill.

\section{MOBIVAS in the context of relevant research and standardization activities}

To facilitate the development of portable, adaptable and functionality-rich services for future mobile systems and networks, various efforts have been undertaken by standardization work groups and fora towards the introduction of open networkindependent interfaces. These interfaces offer transparent access to network functionality (e.g., call control, location information) to $3^{\text {rd }}$ party applications, thus enabling independent application vendors to develop advanced, networkindependent services with standard software engineering tools and general-purpose programming languages. This way, the service creation market is accessible to independent software vendors.

Examples of such efforts are the following:

- Parlay. Parlay is an API that provides $3^{\text {rd }}$ party software developers access to network functionality and allows them to control a selected range of network capabilities [16].

- JAIN [17]. JAIN is a set of Java APIs that aim to enable the rapid development of next generation telecommunications services on the Java 
platform. The JAIN family of specifications includes a wide range of APIs from Java interfaces to network protocols (TCAP, MAP, SIP, H.323) to high level interfaces to be used by applications for access to network functionality.

- OSA. OSA is an API defined by 3GPP aiming to open 3G mobile networks to $3^{\text {rd }}$ party developers [18].

The co-operation of the various standardization bodies results to the streamlining of the different specifications with each other. Notably, the OSA approach is largely based on Parlay and the JAIN external APIs are streamlined with Parlay. These specifications share a common philosophy: services in telecommunication networks should not be standardized (besides a very small number of exceptional cases). Standardization only specifies generic-reusable service features. Naturally, these features are available through open interfaces, so that they can be re-used by 3 rd parties (not only network operators and equipment vendors) to build a wealth of services that take advantage of network-provided functionality, while being network independent.

It is worth noting the difference in scope between MOBIVAS and the abovementioned specifications. MOBIVAS is a service management platform, which aims to be application independent and therefore does not interact directly or provide any interface to $3^{\text {rd }}$ party services, like OSA, Parlay and JAIN do. On the contrary, the MOBIVAS framework aims to complement these approaches.

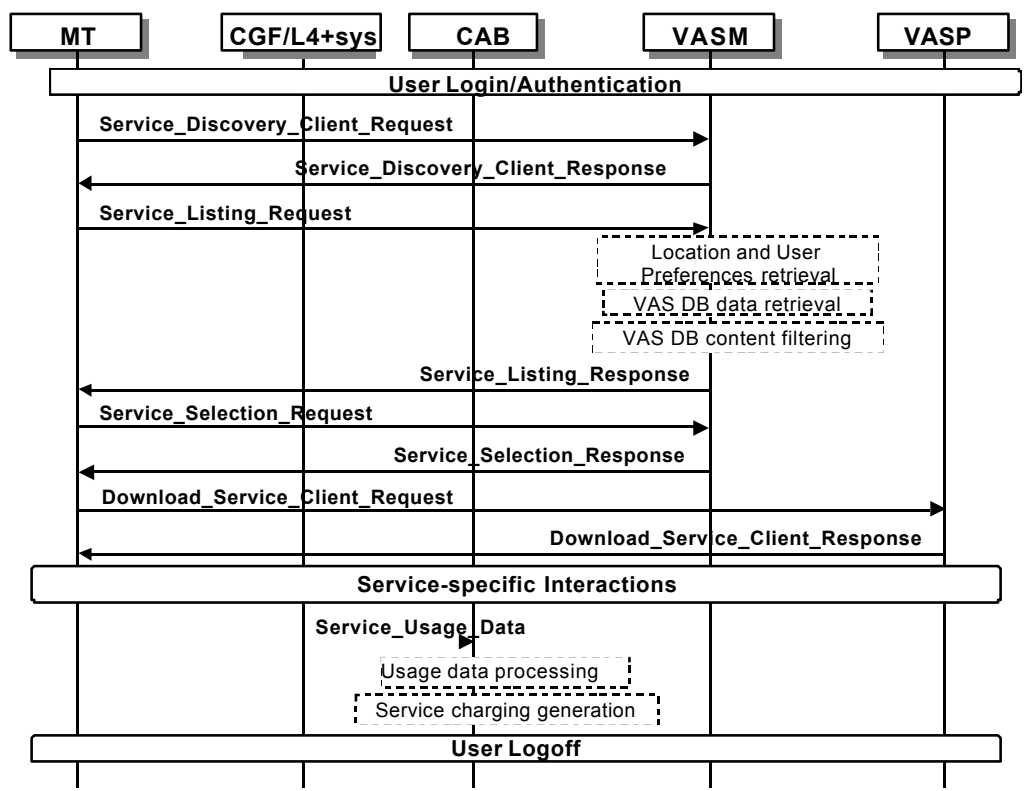

Figure 8. Message sequence diagram for an example platform access session

Network openness, ease of service development and the resulting availability of a large number of services that may be attractive to end users is not adequate for $3 \mathrm{G}$ services to be successful in the market. Sophisticated service management is 
required, so that the available services are quickly and efficiently discovered byand optimally provisioned to end users, while service consumption charges are calculated in flexible ways that make service usage more attractive.

Several significant research efforts have been performed in the area of software platforms for service provisioning in telecommunication and converged environments. IST projects TEQUILA [19], MONASIDRE [20] and INTERNODE [21] are undertaking issues related to lower-level (related to MOBIVAS) aspects of service provisioning in IP and telecommunication networks, like QoS negotiations, resource management and service interworking in multi-domain contexts. The IST VESPER [22] project addresses issues related to VHE provision (e.g., user profiling, roaming across providers, session management). Projects MESH and FRIENDS of the Gigaport initiative [23], have introduced service management platforms based on the TINA model, thus encouraging the development of services by means of existing components (unlike MOBIVAS, where the requirements on service developers are minimal). This approach greatly facilitates software re-use, while imposing certain constraints to service development (services should be created in a specific way).

\section{Summary - Conclusions}

This paper investigated issues related to service management in the new world of $3 \mathrm{G}$ mobile communications and the emerging requirements that make this task significantly more complicated that in the past. An advanced service management and provision framework was introduced as a solution to these issues, aiming also to be complementary and value-adding to relevant standardization activities. In particular, the architecture and functionality of the central platform component, the VASM, was presented in more detail and the implementation of a prototype of the software platform, developed in the context of the MOBIVAS project was briefly described.

\section{Acknowledgments}

Work presented in this paper has been performed in the framework of the project IST MOBIVAS, which is partly funded by the European Community. The Authors would like to acknowledge the contributions of their colleagues from Thales Communications, Hellenic Telecommunications Organisation, NEC Europe LTD, University of Athens, Ecole Nationale Supérieure des Télécommunications, Technical University of Berlin, OTE Consulting, UNIS, IDATE, Innovators. Moreover, the authors would like to particularly acknowledge the valuable contribution of Mr. Spyros Pantazis and Mr. Dimitris Alexandrou to the implementation of the VASM prototype.

\section{References}

[1] N. Houssos, M. Koutsopoulou, S. Schaller, "A VHE architecture for advanced value-added service provision in 3 rd generation mobile 
communication networks" GLOBECOM2000 Workshop on Service

Portability, December, 2000, San Francisco, USA.

[2] UMTS Forum Reports \#8 - \#11, publicly available from http://www.umtsforum.org/.

[3] The UMTS Portal and its Evolving Value Chain, Jorge M. Pereira, INFSO E 4, European Commission at KCGlobal Mobile Portals Europe, 29 March 2001, London, UK, available from http://www.cordis.lu/ist/ka4/mobile/.

[4] 3G TS 22.101: "Services Principles".

[5] 3G TS 22.105: "Services and Service Capabilities".

[6] 3G TS 23.002: "Network architecture".

[7] 3G TS 23.060: "General Packet Radio Service (GPRS); Service description; Stage 2".

[8] 3G TS 23.057: "Mobile Execution Environment (MExE)".

[9] M.Koutsopoulou, C. Farmakis, E. Gazis, "Subscription Management and Charging for Value Added Services in UMTS Networks", IEEE VTC2001, May 2001, Rhodes, Greece.

[10] M.Koutsopoulou, E. Gazis, A. Kaloxylos, " A Novel Billing Scheme for UMTS Networks", International Symposium on 3rd Generation Infrastructure and Services, Athens, Greece, July 2001.

[11] V. Gazis, M. Koutsopoulou, C. Farmakis, A. Kaloxylos, "A Flexible Charging and Billing Approach for the Emerging UMTS Network Operator Role", ATS 2001, Seattle, Washington, USA, April 2001.

[12] Layer 4 Switching: An Overview, http://www.enterasys.com/technologies/smartswitch-router.

[13] M. Schldger, B.Rathke, S. Bodenstein, A. Wolisz, "Advocating a Remote Socket Architecture for Internet Access using Wireless LANs", Mobile Networks \& Applications, vol. 6, no. 1, pp. 23-42, January 2001, Special Issue on Wireless Internet and Intranet Access, Kluwer Academic Publishers.

[14] MOBIVAS deliverable 4.4.1'Implementation of the End User Terminal Platform".

[15] RFC 3084, "COPS Usage for Policy Provisioning (COPS-PR)”, K. Chan, J. Seligson, D. Durham, S. Gai, K. McCloghrie, S. Herzog, F. Reichmeyer, R. Yavatkar, A. Smith, http://www.ietf.org/rfc/rfc3084.txt

[16] Parlay Group, "Parlay API Spec. 2.1", July 2000, available from http://www.parlay.org/specs/index.asp.

[17] J. Keijzer, D. Tait, R.Goedman, "JAIN: A new approach to services in communication networks", IEEE Communications Magazine, January 2000.

[18] 3G TS 23.127: "Virtual Home Environment".

[19] IST TEQUILA project, http://www.ist-tequila.org/.

[20] IST MONASIDRE project, http://www.monasidre.com/.

[21] IST INTERNODE project, http://www.mobile-ip.de/ crist/Data/.

[22] IST VESPER project, http://vesper.intranet.gr.

[23] Gigaport initiative, http://www.gigaport.nl. 